\title{
SYNOPSIS OF A THESIS
}

\section{Linear optics of the pseudophakic eye}

\section{GE MacKenzie*}

Optometric Science Research Group, Department of Optometry, University of Johannesburg, PO Box 524, Auckland Park, 2006 South Africa

< gmk@na.rau.ac.za >

That the eye is essentially a first-order optical instrument is evidenced by the success Gaussian optics has met with in optometry and ophthalmology. An unfortunate consequence of this approach is that a brief review of the literature on the topic of intraocular lens power calculation gives one the impression that the character of such a lens is described fully by its dioptric power. This is not so. Indeed, the idea that a thin refracting interface can somehow embody the optical character of the thick intraocular lens can, and in many ways has, limited the scope of intraocular lens power formula.

The purpose of this thesis is to apply the methods of linear algebra to the investigation of the first-order optical character of the stigmatic and astigmatic pseudophakic eye. This work attempts to lay a solid foundation for the study of the pseudophakic eye in the context of firstorder astigmatic optics.

While the majority of concepts and results of this thesis are directly applicable to the study of the pseudophakic eye, an attempt has been made to ensure that the methods outlined in this work may be applied to the study of optical systems in the broader context of first-order optics.

Central to this work are the members of the non-abelian symplectic group $S p(2 n)$ under the operation of conventional matrix multiplication. The elements are even-dimensional, non-singular symplectic matrices with unit determinant which are referred to here as ray transferences. These matrices act on the members of evendimensional vector spaces so as to preserve a particular skew-symmetric, non-degenerate bilinear pairing referred to as the symplectic form. The laws that govern the operation of these matrices, the three symplectic relations, flow naturally from the structure of the symplectic group. From the ray transference four fundamental properties of an optical system may be defined, the dilation $\mathbf{A}$, the disjugacy $\mathbf{B}$, the divergence $\mathbf{C}$ and the divarication $\mathbf{D}$. A number of additional optical properties can be derived from the fundamental properties. Examples of derived properties include the dioptric power $\mathbf{F}$, the negative of the divergence $\mathbf{C}$ and refractive state $\mathbf{F}_{0}$.

The ray transference is used here in the derivation of a set of new intraocular lens formulae for the pseudophakic eye. These formulae are entirely general, working equally well in both stigmatic and astigmatic pseudophakic eyes in which additional (possibly astigmatic) intraocular devices may already be present. Formulae for both distant and near objects are provided. The constraints under which the divergence of a thick (possibly bitoric) intraocular lens is conserved despite changes in the lens are investigated. Furthermore, the constraints under which the refractive state of the pseudophakic is conserved in spite of changes in the thick intraocu-

\footnotetext{
* DPhil(RAU)

This is a synopsis of a thesis submitted in fulfilment of the requirements of the degree Doctor Philosophiae in Optometry in the Faculty of Science at the Rand Afrikaans University. Promoter: Professor WF Harris, Degree conferred on 3rd December 2004
} 
lar lens are investigated. We find that there exist an infinite number of thick intraocular lenses that will produce a given refractive outcome, say emmetropia, in the pseudophakic eye.

The basic theory of matrix differentiation with respect to a scalar variable is utilized in the study of the changes in the optical character of the pseudophakic eye following axial translation of a variety of intraocular lens systems. A novel method of representing the changes in the stigmatic and antistigmatic properties of refraction on account of axial translation and rotation of a toric intraocular lens in the astigmatic eye is presented and numerical examples are provided. The analysis permits the calculation of the ideal axial lens position and orientation in the astigmatic pseudophakic eye. Such methods will prove increasingly important in refractive data analysis, particularly in light of the development of continuously adjustable intraocular devices.

\section{Summary of the important findings and results of this thesis}

- Formulae for the determination of the optical properties of thick and thin intraocular lenses are derived in terms of the four $2 \times 2$ fundamental properties and the blur, size, spread and directional coefficients of the pseudophakic eye.

- The formulae derived in this thesis are entirely general, working equally well in both stigmatic and astigmatic eyes in which additional astigmatic intraocular devices may be present.

- The formulae derived in terms of the pseudophakic eye's distant blur, size, spread and directional coefficients are special cases of the formulae for near objects. Furthermore, the formulae derived in terms of the blur, size, spread and directional coefficients are special cases of the formulae derived in terms of the $2 \times 2$ fundamental properties.

- The theoretical basis of the A-constant used in some intraocular lens power formulae is derived here and expressed as a relationship between the derived properties of an eye arbitrarily partitioned into an anterior and posterior part.

- The differential analysis presented in this thesis may be used to investigate the potential for restoring accommodation in the pseudophakic eye following the implantation of a variety of so-called 'accommodating intraocular devices'.

- Methods that allow one to investigate the optical effects of rotation and translation of intraocular lenses are examined. These methods may be used to predict accurately the ideal axial position and orientation of toric intraocular lenses following surgical implantation. 